\title{
Inactivity and Inflammation in the Critically Ill Patient
}

\author{
Chris Winkelman, $\mathrm{RN}, \mathrm{PhD}$
}

Frances Payne Bolton School of Nursing, Case Western Reserve University, 10900 Euclid Avenue, Cleveland, OH 44106, USA

The largest organ in the human body, by weight, is skeletal muscle [1]. Muscle is one of the most adaptable organs in people; it is characterized by a continuous cycle of remodeling. Muscle responds quickly to use and disuse by altering diameter, length, types of contractile fibers, and vascular supply. When muscles are not used, as with bed rest, atrophy occurs [2-4]. Elderly patients, with muscle mass reduced from aging, are particularly vulnerable to muscle dysfunction from disuse $[5,6]$.

Bed rest is prescribed commonly in the ICU $[7,8]$. Bed rest can mean complete immobilization; this model is used for deconditioning and disuse studies but is less common clinically. In the ICU, an order for bed rest more typically prohibits weight-bearing mobilization (ie, standing, chair-sitting) and maneuvers that include active muscle contractions, such as dangling (ie, sitting at the edge of the bed without weight-bearing) or isotonic exercise. Even when bedrest does not specifically prohibit repositioning or range of motion, patients in the ICU may experience significant inactivity. Evidence suggests that turning and repositioning in the ICU happens in intervals greater than 2 hours, even when patients are hemodynamically stable $[9,10]$. In the hospital, ambulation and turning are among the most commonly missed nursing interventions [11].

Bed rest is not benign. It has significant physiologic effects on other organ systems that can contribute to muscle dysfunction. Cardiovascular effects of bed rest include a decrement in plasma volume, orthostatic instability, and decreased venous flow in the lower extremities in healthy volunteers [12]. Laying supine during bed rest is associated with a decrease in lung volumes and changes in ventilation and perfusion that contribute to hypoxia through atelectasis, aspiration, and pneumonia in both healthy and intubated patients [13-15]. Bed rest can lead to pressure ulcers and skin breakdown

E-mail address: chris.winkelman@case.edu 
from shearing forces; open wounds may preclude mobilization during treatment and healing $[16,17]$. Inactivity during bedrest results in bone loss, with increased calcium excretion, altering electrolyte balance needed for normal muscle function [18]. Insulin sensitivity is decreased, and hyperglycemia results when skeletal muscles are at rest [4]. Immobilization can result in joint contractures and peripheral nerve injury [19]. Changes in the autonomic and cognitive nervous systems can occur with prolonged bedrest, making mobilization hazardous [20].

Although the effects of bed rest and inactivity have been investigated systematically for more than 40 years, the physiological causes of adverse outcomes are not clear. It is believed to be a combination of several factors, including the inflammatory cascade. In at least two chronic diseases, heart failure (HF) and chronic obstructive pulmonary disease (COPD), skeletal muscle wasting is a common concurrent clinical finding. Low-grade chronic inflammatory dysregulation, not deconditioning, in HF and COPD appear to contribute to myopathy [21,22]. Inflammatory dysregulation also is thought to contribute to muscle loss in elders [6].

This article describes the role of two proinflammatory processes that contribute to muscle dysfunction. Activity is explored as a process that influences inflammatory regulation and muscle function. The utility of activity and mobility therapy for ICU patients, especially nonsurgical patients, is not well-established [23].

\section{Inactivity and muscle dysfunction}

Deconditioning is defined as the multiple changes in organ systems that are caused by inactivity [24]. Heart, lung, and muscle deconditioning have been studied extensively. In normal, healthy subjects, there are immediate neuromuscular effects related to inactivity. For example, within 4 hours of bed rest, muscles deteriorate [3]. Sarcomeres are reduced, shortening muscle fibers and total muscle length. There is a loss of contractile force resulting in decreased strength. With a daily loss of $1.3 \%$ to $3 \%$ of muscle strength during immobility, a $10 \%$ reduction in postural muscle strength can occur after 1 week of complete bed rest in healthy volunteers [25]. Deconditioning can lead to disuse syndromes that are difficult to reverse. For example, contractures can begin to form after 8 hours of immobility. Inactivity has direct effects on skeletal muscles. It also affects other systems, contributing to myopathy.

Changes in skeletal musculature, specifically quadriceps strength, have been associated with reduction in lung function [26]. Inflammatory disease can cause diaphragmatic contractile dysfunction, contributing to respiratory failure [27]. Diaphragmatic endurance also can be reduced by unloading or resting the diaphragm during prolonged mechanical ventilatory support [27,28]. Diaphragmatic changes in strength or endurance from myopathic changes caused by inflammation and deconditioning may contribute to prolonged mechanical ventilation in ICU patients. 
Bed rest over 48 hours in healthy volunteers results in decreased perfusion and risk for hemodynamic instability with posture changes through decrements in plasma volume, electrolyte loss, and heart rate and blood pressure changes that occur within 48 hours of bed rest [18]. These changes in blood flow and perfusion can impair circulation and put skeletal and smooth muscles at risk for ischemia and injury. Even passive movement, as with range of motion, is thought is improve circulation locally and systemically [29].

There are important bidirectional interactions between the autonomic nervous system and cellular signaling [30]. The parasympathetic system has been identified as a promoter of anti-inflammation [31]. Stimulation of the efferent vagus nerve can inhibit inflammation [32]. It may be that inactivity contributes to inflammation by decreasing vagus nerve activity through altering respiratory patterns and the absence of abdominal and gluteal muscle contractions.

Bed rest is a subtle form of sensory deprivation that can alter mood and anxiety as well as sleep patterns. Anxiety, hostility, depression, and disturbed sleep patterns have been demonstrated in studies of normal, healthy men during several weeks of bed rest [33]. These changes may contribute to delirium and exacerbation of dementia in the ICU. Hospitalized patients who are confused and agitated are more likely to be sedated and restrained, contributing to immobility and subsequent complications [34].

Skeletal muscle dysfunction is linked to a subjective sensation of fatigue $[35,36]$. For example, cytokines mediate sickness syndrome, characterized by fatigue and malaise [37,38]. Even among healthy individuals, fatigue can lead to self-limitation of activity and mobility [6]. The subjective sense of fatigue may contribute unwillingness to participate in rehabilitative interventions, prolonging bed rest and promoting deconditioning and a cycle of fatigue and inactivity.

Thus, inactivity can have multiple direct and indirect effects on musculature with implications for the patient in the ICU. There direct effects related to disuse and deconditioning and indirect effects from alterations in ventilation, circulation, the central nervous system (including autonomic and cognitive changes) and feeling of fatigue. Among hospitalized patients, especially elderly patients, loss of function results in discharge to longterm or rehabilitative care, adding to the physical, emotional, and economic costs of a life-threatening illness $[25,39,40]$. The mechanisms leading to muscle decay from inactivity are not understood completely. Two interactive molecular pathways have been implicated: oxidative stress and selected inflammatory cytokines. These mechanisms are described in the next section.

\section{Oxidants, cytokines, and myopathy}

Some evidence suggests that inactivity can contribute to imbalances in the inflammatory response $[41,42]$. Both oxidative stress and proinflammatory 
cytokines have been investigated as potential causes for myopathy during acute and critical illness. It may be that there is a synergy between oxidative stress, inflammatory cytokines, and inactivity such that atrophy is accelerated or compounded. If true, then there are important implications for clinicians in terms of preventative and rehabilitative interventions. The role of oxidative stress and selected cytokines on musculature has been studied in the laboratory and in animal models; supportive data also link activity to changes in inflammatory cellular messengers in healthy patients.

\section{Oxidative stress influences muscle dysfunction and degradation}

Reactive oxygen species (ROS) and nitric oxide (NO) are capable of causing oxidative damage to DNA, lipids, and proteins. Oxidants mediate various processes that contribute to inflammatory damage to the muscles. Examples of ROS include superoxide ion $\left(\mathrm{O}_{2}{ }^{-}\right)$and hydroxyl radicals $\left(\mathrm{OH}^{-}\right)$. Critically ill patients are vulnerable to synthesis of excess oxidants and reduced levels of chemicals that metabolize oxidants.

Oxidative stress, also known as increased oxidant burden, is thought to play a role in the pathophysiology of muscle dysfunction [43-45]. ROS react with the lipids in myocyte cell membranes. Lipid peroxidation, in turn, releases toxins and arachidonic acid derivatives that can damage cell membranes further, inactivate membrane receptors and membrane-bound enzymes, and alter ion channel responses. In myocytes, this degradation process also leads to modification of numerous intracellular signaling pathways in vitro, including activation of the nuclear factor kappa-b (NF- $\kappa \mathrm{B})$ pathway [46]. Products from cellular destruction by ROS provide a positive feedback loop, generating more ROS [47]. At the same time, ROS interact with cytokines and other intercellular molecules associated with muscle degradation $[48,49]$. In addition, ROS appear to stimulate central and peripheral sympathetic nervous systems in both tissue and animal models, contributing to autonomic dysfunction [50,51].

$\mathrm{NO}$ and its derivatives also are formed in the contracting muscle by the enzyme NO synthase (NOS). At low rates, NO regulates intra-and intercellular signaling. Inflammatory stimuli can stimulate expression of inducible NOS (iNOS) by muscle cells, adding to oxidant burden [52]. NO also enhances intravascular blood flow through vasodilation and may have a role to play in reducing muscle fatigue by improving blood flow [27].

There is a strong link between oxidant stress and contractile function in skeletal muscles. Muscles are a source of oxidant synthesis, especially during exercise [53]. Both ROS and NO are necessary for normal skeletal muscle contraction. Under basal conditions, low levels of ROS and NO enhance contractile force [54]. During fatiguing exercise, contractile force is reduced by ROS. During mechanical unloading as with mechanical ventilation, there is an experimental increase in oxidant production [55]. The phenomenon of mechanical loading may help explain why daily weaning is useful in weaning 
success; the weaning trial reverses the effects of mechanical unloading during ventilatory support. Importantly, the depletion of ROS also depresses contractile force [27]. It also may be that therapeutic mobility, mechanical loading, is beneficial to contractile regulation in skeletal muscles if it promotes synthesis of beneficial levels of oxidants needed for cellular signaling and maintenance of myofilament structure.

Antioxidant scavengers and enzymes such as superoxide dismutase (SOD), catalase, and glutathione peroxidase (GPX) reduce oxidants by combining with them. Antioxidants are reduced during disease states common to ICU patients such as COPD, acute lung injury, atherosclerosis, and acute renal failure $[43,56,57]$. These antioxidants are produced by skeletal muscles [53]. Repetitive training can increase antioxidant synthesis and activity in cells and healthy people $[58,59]$.

\section{Selected cytokines influence muscle dysfunction and degradation}

Several cytokines have received attention as potential biomarkers of critical illnesses and adverse outcomes. Several of these cytokines overlap with investigations in exercise-activity physiology. Cytokines with importance to both critical illnesses and in exercise/activity are tumor necrosis factor $\alpha$ (TNF- $\alpha$ ) and interleukins (IL) $-1,-6$, and -10 .

Any cell with a nucleus can produce TNF- $\alpha$. Along with ROS, TNF- $\alpha$ induces NF- $\kappa \mathrm{B}$, promoting skeletal muscle degradation. Direct exposure to TNF- $\alpha$ results in decreased muscle cell contraction in the laboratory [60]. In a model to explain cachexia, Reid and Li [44] suggested the interaction between ROS and proinflammatory cytokines like TNF- $\alpha$ are synergistic, perhaps indicative of a pathological positive feedback loop, which downregulates repair of damaged muscle tissue. Thus, it is not simply direct suppression of muscle activity that leads to dysfunction in the presence of TNF- $\alpha$, but a decrement in repair and/or increment in apoptosis that results in cytokine-mediated muscle decay. In healthy individuals, increments in TNF- $\alpha$ are variable in response to exercise [61-63]. There is some indication that TNF- $\alpha$ is highest when oxidative stress is present during exercise [62]. Serum TNF- $\alpha$ also reduces the levels of some antioxidants in skeletal muscles [44].

IL-1 is a superfamily with four related ligands: IL-1 $\alpha$, IL-1 $\beta$, IL-18, and IL-1Ra (receptor antagonist). IL-1 Ra acts as an inhibitory molecule, antagonizing the inflammatory effects of IL-1. IL-18 contributes to the induction of reactive oxygen species, $\mathrm{TNF} \alpha$, and other pro-inflammatory molecules, including interferon gamma. The biologic actions of Il-1 $\alpha$ and IL-1 $\beta$ are similar, but only IL- $1 \beta$ is secreted vascularly; it is more likely to mediate systemic inflammation [64]. Like TNF- $\alpha$, IL-1 $\beta$ is an upstream proinflammatory cytokine, stimulating the synthesis of various factors including enzymes that produce prostaglandins, adhesion molecules, NO, and ROS [65]. In health, spontaneous production of IL-1 $\beta$ is nearly nonexistent 
[64]. In the presence of strenuous exercise in athletes, plasma IL-1 $\beta$ values have been reported to increase and to decrease $[61,66]$. One interesting finding in a study of human patellar fibroblasts stimulated by IL-1 $\beta$ suggests that small-magnitude stretching is anti-inflammatory (with reduced cyclooxygenase and prostaglandin synthesis under the influence of IL-1 $\beta$ ), whereas large-magnitude stretching was proinflammatory with increased levels of inflammatory products stimulated by IL-1 $\beta$ [67]. In cell cultures of chondrocytes, cyclic tensile strain of a low magnitude antagonizes TNF- $\alpha$ and promotes IL-10, indicating that small-to-moderate stretch may promote anti-inflammation response in articular cartilage [68]. In this same laboratory, high levels of strain increased inflammation [69].

IL-6 has a wide range of biologic activity [70]. IL-6 is synthesized by immune cells and by skeletal muscle cells, adipocytes, endothelial cells, and intestinal epithelial cells [71]. Similar to TNF- $\alpha$ and IL-1 $\beta$, IL-6 is released early in the inflammatory cascade. Unlike TNF- $\alpha$, IL-6 increases myosin and appears to have a role in maintaining myocyte energy supply during exercise [72]. Muscle contraction induces the production and release of IL-6 into the plasma in large amounts, up to a 100 fold increase over baseline values in various human studies [61]. The synthesis of IL-6 from exercise is independent of production from the TNF- $\alpha$ pathway [61]. During exercise, IL- 6 also is synthesized and placed into circulation by peri-tendon and adipose tissues. Even brief, low-intensity exercise of a small muscle group-unilateral wrist flexion for 10 minutes - in healthy young adults dramatically increased IL-6 systemically $(+139 \%,+/-51 \%)$ [73].

In addition to proinflammatory properties, high levels of IL-6 act to stimulate the appearance of anti-inflammatory cytokines in plasma, including IL-10 and IL-1Ra [74]. Muscle-derived IL-6 reduces TNF- $\alpha$ production, interrupting the muscle degradation by means of myosin destruction. Suppression of proinflammatory cytokines with IL-6, especially the powerful IL-1 $\beta$ and TNF- $\alpha$, may benefit critically ill patients. In one study of untrained but healthy women (average age of 37 years), walking for 30 minutes at submaximal effort increased IL-6 immediately after exercise, with a return to baseline 1 hour after ambulation [75].

IL-10 is an anti-inflammatory cytokine, originally identified by its ability to turn off cytokine production by $\mathrm{T}$ cells. Investigations have shown that IL-10 inhibits synthesis of IL-1 $\beta$, IL-6, TNF- $\alpha$, reactive oxygen intermediates, and other proinflammatory factors, suppressing multiple immune responses through individual actions on several cell types [76]. After exercise, high circulating levels of IL-6 are followed by increased production of IL-10 [61]. IL-10 is increased in plasma even after a single bout of limited exercise-30 contractions of elbow flexors in untrained, healthy males [77]. Prolonged, strenuous activity in healthy males resulted in an immediate 27 fold increase in IL-10 [66].

Investigations of healthy men and women suggest that exercise causes anti-inflammation by inducing both IL-10 and IL-6 and inhibiting 
TNF- $\alpha$ and IL-1 $\beta$. The cellular messengers, IL-6 and IL-10, are implicated in maintaining muscle function in stretch and some types of exercise. Although low levels of exercise induce ROS, the amount of ROS may not be damaging. Repetitive exercise can improve levels of antioxidant scavengers in muscles over time. Clinically, training and rehabilitation in cardiac and pulmonary disease promotes function, even among the frail elderly population [78-80].

\section{Oxidants, cytokines, and activity}

There are few published data about the effect of exercise activities on oxidants or cytokines in critically ill patients [10]. Some clinicians may believe that ICU patients may not be able to perform activity or mobility therapy at sufficient intensity to produce effects locally (ie, within the muscle) or systemically. Yet studies of healthy adults suggest that low and moderate levels of activity produce measurable physiological changes measured in muscle biopsies and plasma values. In the ICU population, demonstrating similar changes will be challenging. Data from studies of patients with chronic inflammatory diseases such as heart failure (HF), COPD, and rheumatoid arthritis provide information that activity is beneficial and does not exacerbate inflammation. These studies are summarized.

One pilot study examined the changes of plasma cytokines in hemodynamically stable patients who experienced an average of 10 days of mechanical ventilation. Data suggested that 20 minutes of sustained activity in bed (repositioning and range of motion activities) did not alter plasma levels of IL-6 or IL-10 [81]. This sample was small $(\mathrm{n}=10)$ and focused on chronically, critically ill adults, so findings may not be applicable to all ICU adults.

Several studies have examined the influence of activity on serum cytokines in the presence of HF. At baseline and after 6 months, serum samples and vastus lateralis muscle biopsies were obtained. Exercise training in 10 patients who had HF reduced the skeletal muscle expression of TNF- $\alpha$, IL-1B, IL-6, and inducible NOS (iNOS), while serum levels of the cytokines remained unchanged [82]. In another study, 24 patients who had stable HF participated in a 12-week program of physical training in a randomized, crossover design. Physical training produced a reduction in plasma TNF- $\alpha$ and IL-6 at the end of 12 weeks of training [83]. In this study, no significant reduction in cytokines was found after similar physical training of normal controls. In a third study, 15 men who had stable HF underwent aerobic exercise training for 12 weeks. At the end of the program, subjects experienced a decrease in plasma IL-6 and a trend toward increased skeletal muscle fiber thickness compared with values at the beginning of the program [84]. A fourth study demonstrated reductions in TNF- $\alpha$ and IL-6 in HF subjects over 6 weeks, while another study indicates no change in the same two 
cytokines after 4-month endurance training in similar HF subjects $[85,86]$. One study demonstrated increased gene expression for antioxidative enzymes after physical training in $\mathrm{HF}$ patients without concurrent increase in NO or prostaglandins in intravascular cells [87]. This series of studies, along with data from healthy controls who exercise [88], indicates that physical training over time does not exacerbate proinflammatory cytokines in patients who have HF.

In a related study, 28 patients with coronary artery disease were followed over a 12-week aerobic rehabilitative program. Training resulted in a decrease in IL- $1 \beta$ and IL- 6 by about $30 \%$ and an increase in IL- 10 by over $50 \%$. These changes were accompanied by a reduction in C-reactive protein, with a $46 \%$ reduction in the number of subjects in a high-risk category for adverse cardiac advents [89]. Low levels of IL-10 predict decreased activity and increased angina in patients who have unstable angina, but it is not clear whether low activity causes low IL-10, or whether low IL-10 results in decreased activity [90].

Among studies of patients who have COPD, exercise and training have demonstrated an ability to alter oxidant burden. In one study, the antioxidant citrate synthase in the vastus lateralis was increased at the end of a 12-week endurance program for patients who had moderate COPD [91]. Other studies have not confirmed an increase in antioxidant formation as a result of structured exercise. In two studies of COPD patients, initially and immediately after exercise, oxidant stress was increased when glutathione peroxidase did not increase [92,93]. The study protocols, however, were not clear in whether plasma oxygen levels were monitored or if additional oxygen was provided to COPD patients during exercise to prevent hypoxia. Activity or mobility in critically ill patients may initiate hypoxia; hypoxia is a source of increased oxidants [94,95]. Koechlin and colleagues [96] suggest that arterial oxygenation is key to reducing oxidative stress in patients who have COPD. In a sample of 10 subjects who had COPD, plasma levels of products of oxidative stress were increased significantly over similar products formed by seven controls at 6 hours after one episode of quadriceps endurance activity. Nonenzymatic antioxidants and inflammatory cytokine levels were unchanged by exercise immediately after and up to 48 hours after quadriceps endurance activity [97]. In this study, proinflammatory cytokines in plasma remained unchanged up to 6 hours after one episode of exercise. The authors concluded that local exercise-induced oxidant stress did not lead to increased systemic markers of inflammation over baseline in COPD patients or controls.

Disease processes other than HF and COPD have been used to investigate the effect of exercise on inflammatory markers. Three consecutive days of exercise in participants with sickle cell anemia, characterized by both chronic and acute inflammatory markers, did not affect serum IL-6 levels but decreased TNF- $\alpha$ in sickle cell patients on day 3 when compared with the first and second day of exercise [98]. In Parkinson's disease, patients 
who had evidence of baseline chronic inflammation performed cyclic exercise resulting in increased plasma IL-10 but unaltered IL-1 $\beta$ or IL-6. Concurrently, patients reported enhanced motor skills and improvement in mood [99]. In another study examining low levels of activity in the presence of an inflammatory disease, 93 patients with rheumatoid arthritis were randomized to either a rehabilitation or control group. The rehabilitation group experienced increased quadriceps sensorimotor function and decreased lower limb disability without exacerbating pain or disease activity. Specifically, IL-6 did not increase immediately after the first period of physical training, mid-way through the fourth week intervention, or at the end of the intervention when compared with the nonintervention group. Results (including unaltered serum IL-6 levels) were sustained at a 6-month follow-up after completion of rehabilitation activities [100]. In this study, decrements of IL-6 were not evaluated, but a report of baseline values of $37 \mathrm{pg} / \mathrm{mL}$ at rest compared with $27 \mathrm{pg} / \mathrm{mL}$ after rehabilitative activity suggests a decreasing value.

These studies seem to indicate activity modulates proinflammatory processes and/or does not exacerbate inflammatory molecules in the presence of underlying systemic disease. The exception may be the presence of a transient increase in oxidative products during and immediately after strenuous activity. Avoiding hypoxia and fatiguing repetitions appears to be important to minimizing the synthesis of inflammatory molecules, especially ROS. Further, mild stretch (rather than extreme stretch) that is common in active and passive range-of-motion activity may improve inflammatory disequilibrium. Most studies reviewed in this section used moderate-to-strenuous levels of exercise without adverse systemic effect. Exercise activities have beneficial effects in terms of muscle strength, endurance, and overall function in various inflammatory diseases $[61,101]$.

In the ICU, low levels of activity and mobility therapy could be implemented for selected patients. Low levels of activity have the potential to interrupt myopathy directly. Activity in ICU patients also may improve capillary dynamics, reducing the synthesis and distribution of proinflammatory messengers. For example, venous stasis allows local oxidants and cytokines to accumulate, with the potential for spillover into systemic circulation and effects [102]. Intermittent mobility therapy and active range of motion may increase both NO formation and pulsatile blood flow in vasodilated vessels, diluting local accumulation of proinflammatory factors such that a critical mass inducing systemic affects does not occur. Even the briefest interventions from repositioning and passive range of motion may reduce capillary compression and local low-flow states. Further investigation is needed to determine if activity and mobility therapy could influence the functional outcomes of critically ill patients positively without exacerbating inflammatory processes. Determining the dose of activity-frequency, duration, and intensity-may be guided by using inflammatory biomarkers such as ROS and cytokines. 


\section{Summary}

It is common for patients in the ICU to experience bed rest, sometimes for prolonged periods. Bed rest may be a natural consequence of illness and beneficial for recovery. Inactivity, however, especially during prolonged illness, contributes to muscle loss and decrements in functional status. Bed rest among ICU patients has had limited investigation. The duration and body positions that produce optimal outcomes related to a prescription of bed rest are not known. The mode, duration, and frequency of activity have not been investigated adequately among patients in the ICU. Examining the effects on cytokines or ROS after low-to-moderate activity in critically ill subjects may be one way to guide the dose of bed rest or prescribe therapeutic mobility in the ICU. Examination of the role of cytokines and oxidants may help explain the early, adverse effects of bed rest and provide information as biomarkers that guide activity during critical illness. Understanding how and when to implement bed rest and activity has potential to improve outcomes for ICU patients.

\section{References}

[1] Guyton AC, Hall JE. Textbook of medical physiology. 11th edition. Philadelphia: Saunders Elsevier; 2006.

[2] Sargeant AJ, Davies CTM, Edwards RHT. Functional and structural changes after disuse of human muscle. Clin Sci (Colch) 1977;52:337-42.

[3] Kasper C, Talbot LA, Gaines JM. Skeletal muscle damage and recovery. AACN Clin Issues 2002;15(2):237-47.

[4] Convertino VA, Bloomfield SA, Greenleaf JE. An overview of the issues: physiological effects of bed rest and restricted physical activity. Med Sci Sports Exerc 1997;29(2):187-90.

[5] Creditor MC. Hazards of hospitalization of the elderly. Ann Intern Med 1993;118(3): $219-23$.

[6] Bautmans I, Njemini R, Lambert M, et al. Circulating acute-phase mediators and skeletal muscle performance in hospitalized geriatric patients. J Gerontol A Biol Sci Med Sci 2005; 60(3):361-70.

[7] Stiller K. Physiotherapy in intensive care: towards an evidence-based practice. Chest 2000; 118(6):1801-13.

[8] Allen C, Glasziou P, Del Mar C. Bed rest: a potentially harmful treatment needing more careful evaluation. Lancet 1999;354:1229-33.

[9] Krishnagopalan S, Johnson EW, Low LL, et al. Body positioning of intensive care patients: clinical practice versus standards. Crit Care Med 2002;30(11):2588-92.

[10] Winkelman C, Higgins PA, Chen Y-JK. Activity in the chronically critically ill. Dimens Crit Care Nurs 2005;24(6):281-90.

[11] Kalisch BJ. Missed nursing care: a qualitative study. J Nurs Care Qual 2006;21(4):306-13.

[12] Harper CM, Lyles YM. Physiology and complications of bed rest. J Am Geriatr Soc 1988; 36(11):1047-54.

[13] Mentzelopoulos SD, Roussos C, Zakynthinos SG. Static pressure volume curves and body posture in acute respiratory failure. Intensive Care Med 2005;31(12):1683-92.

[14] Burns SM, Egloff MB, Ryan B, et al. Effect of body position on spontaneous respiratory rate and tidal volume in patients with obesity, abdominal distention, and ascites. Am $\mathbf{J}$ Crit Care 1994;3(2):102-6. 
[15] Rubin M. The physiology of bed rest. Am J Nurs 1988;88(1):50-6.

[16] Peerless JR, Davies A, Klein D, et al. Skin complications in the intensive care unit. Clin Chest Med 1999;20(2):453-61.

[17] Carlson EV, Kemp MG, Shott S. Predicting the risk of pressure ulcers in critically ill patients. Am J Crit Care 1999;8(4):262-9.

[18] Fortney SM, Schneider VS, Greenleaf JE. The physiology of bedrest. In: Fregky MJ, Blatteis CM, editors. The handbooks of physiology. New York: Oxford University Press; 1996. p. 889-939.

[19] Bergel RR. Disabling effects of inactivity and importance of physical conditioning. A historical perspective. Rheum Dis Clin North Am 1990;16(4):791-801.

[20] Ely EW, Siegel MD, Inouye SK. Delirium in the intensive care unit: an under-recognized syndrome of organ dysfunction. Semin Respir Crit Care Med 2001;22(2):115-26.

[21] Duscha BD, Annex BH, Green HJ, et al. Deconditioning fails to explain peripheral skeletal muscle alterations in men with chronic heart failure. J Am Coll Cardiol 2002;39:1170-4.

[22] Mador MJ, Bozkanat E. Skeletal muscle dysfunction in chronic obstructive pulmonary disease. Respir Res 2001;2(4):216-24.

[23] Szaflarski NL. Immobility phenomena in critically ill adults. In: Clochesy J, Breu C, Cardin S, et al, editors. Critical care nursing. 2nd edition. Philadelphia: Saunders; 1996. p. 1313-34.

[24] Oliver FL. Suggested guidelines for the use of exercise with adults in acute care settings: a computerized key work search. Physiother Can 1998;50(2):127-36.

[25] Topp R, Ditmyer M, King K, et al. The effect of bed rest and potential of prehabilitation on patients in the intensive care unit. AACN Clin Issues 2002;13(2):263-76.

[26] Yende S, Watarer GW, Tolley EA, et al. Inflammatory markers are associated with ventilatory limitation and muscle dysfunction in obstructive lung disease in well-functioning elderly subjects. Thorax 2006;61:10-6.

[27] Smith MA, Reid MB. Redox modulation of contractile function in respiratory and limb skeletal muscle. Respir Physiol Neurobiol 2006;151:229-41.

[28] MacIntyre NR, Epstein SK, Carson S, et al. Management of patients requiring prolonged mechanical ventilation: report of a NAMDRC consensus conference. Chest 2005;128(6): 3937-54.

[29] Hall C, Body L. Therapeutic exercise: moving toward function. Philadelphia: Lippincott; 1999.

[30] Tracey KJ. The inflammatory reflex. Nature 2002;420(19):853-9.

[31] Czura CJ, Tracey KJ. Autonomic regulation of immunity. J Intern Med 2005;257:156-66.

[32] Pavlov VA, Tracey KJ. The cholinergic anti-inflammatory pathway. Brain Behav Immun 2005;19:493-9.

[33] Mallis MM, DeRoshia CW. Circadian rhythms, sleep, and performance in space. Aviat Space Environ Med 2005;76(6 Suppl):B94-107.

[34] Mott S, Poole J, Kenrick M. Physical and chemical restraints in acute care: their potential impact on rehabilitation of older people. Int J Nurs Pract 2005;11(3):95-101.

[35] Aaronson LS, Burman ME. Use of health records in research: reliability and validity issues. Res Nurs Health 1994;17(1):67-73.

[36] St. Pierre BA, Kasper CE, Lindsey AM. Fatigue mechanisms in patients with cancer: effects of tumor necrosis factor and exercise on skeletal muscle. Oncol Nurs Forum 1992;19(3): 419-25.

[37] Corwin EJ. Understanding cytokines part I: physiology and mechanism of action. Biol Res Nurs 2000;2(1):30-40.

[38] Corwin EJ. Understanding cytokines part II: implications for nursing research and practice. Biol Res Nurs 2000;2(1):41-8.

[39] Ceriana P, Delmastro M, Rampulla C, et al. Demographics and clinical outcomes of patients admitted to a respiratory intensive care unit located in a rehabilitation center. Respir Care 2003;48(7):670-6. 
[40] Hirsch CH, Sommers L, Olsen A, et al. The natural history of functional morbidity in hospitalized older adults. J Am Geriatr Soc 1990;38(12):1296-303.

[41] Bruunsgaard $\mathrm{H}$. Physical activity and modulation of systemic low-level inflammation. J Leukoc Biol 2005;78(4):819-35.

[42] Ji LL. Exercise, oxidative stress, and antioxidants. Am J Sports Med 1996;24:520-4.

[43] Langen RCJ, Korn SH, Wouters EFM. Serial review: role of reactive oxygen and nitrogen species (ROS/RNS) in lung injury and diseases. Free Radic Biol Med 2003;35(3):226-35.

[44] Reid MB, Li Y-P. Tumor necrosis factor- $\alpha$ and muscle wasting: a cellular perspective. Respir Res 2001;2:269-72.

[45] Couillard A, Prefaur C. From muscle disuse to myopathy in COPD: potential contribution of oxidative stress. Eur Respir J 2005;26(4):703-19.

[46] Li Y-P, Schwartz RJ, Waddell ID, et al. Skeletal muscle myocytes undergo protein loss and reactive oxygen-mediated NF-kappaB activation in response to tumor necrosis factor alpha. FASEB 1998;12:871-80.

[47] Mishra V, Baines M, Wenstone R, et al. Markers of oxidative damage, antioxidant status and clinical outcome in critically ill patients. Ann Clin Biochem 2005;42(Pt 4):269-76.

[48] Flohe L, Brigelius-Flohe R, Saliou C, et al. Redox regulation of NF-kappa B activation. Free Radic Biol Med 1997;22:1115-26.

[49] Janssen-Heininger YM, Poynter ME, Baeuerle PA. Recent advances towards understanding redox mechanisms in the activation of nuclear factor kappa B. Free Radic Biol Med 2000;28:1317-27.

[50] Campese VM, Ye S, Zhong H, et al. Reactive oxygen species stimulate central and peripheral sympathetic nervous system activity. Am J Physiol 2004;287(2):H695-703.

[51] Danson EJF, Paterson DJ. Reactive oxygen species and autonomic regulation of cardiac excitability. J Cardiovasc Electrophysiol 2006;17(5):S104-12.

[52] Stamler J, Meissner G. Physiology of nitric oxide in skeletal muscle. Physiol Rev 2001;81: 209-37.

[53] Clanton TL, Zuo L, Klawitter P. Oxidants and skeletal muscle function: physiologic and pathophysiologic implications. Proc Soc Exp Biol Med 1999;222(3):253-62.

[54] Reid CL, Perrey C, Pravica V, et al. Genetic variation in proinflammatory and anti-inflammatory cytokine production in multiple organ dysfunction syndrome. Crit Care Med 2002; 30(10):2216-21.

[55] Falk DJ, Deruiseau KC, Van Gammeren DL, et al. Mechanical ventilation promotes redox status alterations in the diaphragm. J Appl Physiol 2006;101(4):1017-24.

[56] Laberge MA, Moore KJ, Freeman MW. Atherosclerosis and innate immune signaling. Ann Med 2005;37(2):130-40.

[57] Simmons EM, Himmelfarb J, Sezer MT, et al. Plasma cytokine levels predict mortality in patients with acute renal failure. Kidney Int 2004;65:1357-65.

[58] Fluck M. Exercise-modulated mitochondrial phenotype: sensors and gene regulation. J Muscle Res Cell Motil 2004;25:235-7.

[59] Ortenblad N, Madsen K, Djurhuus MS. Antioxidant status and lipid peroxidation after short-term maximal exercise in trained and untrained humans. Am J Physiol 1997;272: 1258-63.

[60] Reid MB, Andrade FH, Balke CW, et al. Redox mechanisms of muscle dysfunction in inflammatory disease. Phys Med Rehabil Clin N Am 2005;16(4):925-49.

[61] Petersen AMW, Pederson BK. The anti-inflammatory effect of exercise. J Appl Physiol 2004;98:1154-62.

[62] Vassilakopoulos T, Karatza MH, Katsaounou P, et al. Antioxidants attenuate the plasma cytokine response to exercise in humans. J Appl Physiol 2003;94(3):1025-32.

[63] Pedersen BK. Exercise and cytokines. Immunol Cell Biol 2000;78:532-5.

[64] Dinarello CA. Interleukin-1B. Crit Care Med 2005;33(12):S460-2.

[65] Dinarello C. Interleukin-1 family. In: Thomson AW, Lotze MT, editors. The cytokine handbook. Vol. 2, 4th edition. New York: Academic Press; 2003. p. 643-68. 
[66] Ostowski K, Rohde T, Asp S, et al. Pro- and anti-inflammatory cytokine balance in strenuous exercise in humans. J Physiol 1999;515(Pt 1):287-91.

[67] Yang G, Im HJ, Wang JH. Repetitive mechanical stretching modulates IL-1beta-induced COX-2, MMP-1 expression and PGE2 production in human patellar tendon fibroblasts. Gene 2005;363:166-72.

[68] Long P, Gassner R, Agarwal S. Tumor necrosis factor $\alpha$. Dependent proinflammatory gene induction is inhibited by cyclic tensile strain in articular chondrocytes in vitro. Arthritis Rheum 2001;44(10):2311-9.

[69] Gassner RJ, Buckley MJ, Studer RK, et al. Interaction of strain and interleukin-1 in articular cartilage: effects on proteoglycan synthesis in chondrocytes. Int J Oral Maxillofac Surg 2000;29(5):389-94.

[70] Kishimoto T. Interleukin-6. In: Thomson AW, Lotze MT, editors. The cytokine handbook. Vol. 1, 4th edition. New York: Academic Press; 2003. p. 281-304.

[71] Fink MP. The prevention and treatment of sepsis: Is interleukin-6 a drug target or a drug? Crit Care Med 2006;34(3):919-20.

[72] Steensberg A. The role of IL-6 in exercise-induced immune changes and metabolism. Exerc Immunol Rev 2003;9:40-7.

[73] Nemet D, Hong S, Mills PJ, et al. Systemic vs local cytokine and leukocyte responses to unilateral wrist flexion exercises. J Appl Physiol 2002;93(2):546-54.

[74] Starkie R, Ostrowski SR, Jauffred S, et al. Exercise and IL-6 infusion inhibit endotoxininduced TNF-alpha production in humans. FASEB J 2003;17(8):884-6.

[75] Nieman DC, Nehlsen-Cannarella SL, Fagoaga OR. Immune response to two hours of rowing in female elite rowers. Int J Sports Med 1999;20:476-81.

[76] Ding Y, Fu S, Samarin D, et al. Interleukin-10. In: Thomson AW, Lotze MT, editors. The cytokine handbook, vol. 1. New York: Academic Press; 2003. p. 603-25.

[77] Hirose L, Nosaka K, Newton M, et al. Changes in inflammatory mediators following eccentric exercise of the elbow flexors. Exerc Immunol Rev 2004;10:75-90.

[78] Dolansky MA, Moore SM. Effects of cardiac rehabilitation on the recovery outcomes of older adults after coronary artery bypass surgery. J Cardiopulm Rehabil 2004;24(4):236-44.

[79] Anzueto A. Muscle dysfunction in the intensive care unit. Clin Chest Med 1999;20(2):1-25.

[80] Bartels MN, Whiteson JH, Alba AS, et al. Cardiopulmonary rehabilitation and cancer rehabilitation. 1. Cardiac rehabilitation review. Arch Phys Med Rehabil 2006;87(3 Suppl 1): S46-56.

[81] Winkelman C, Higgins PA, Chen Y-JK, et al. Cytokines in chronically critically ill patients after activity and rest. Biol Res Nurs, in press.

[82] Gielen S, Adams V, Mobius-Winkeler S, et al. Anti-inflammatory effects of exercise training in the skeletal muscle of patients with chronic heart failure. J Am Coll Cardiol 2003; 42(5):869-72.

[83] Adamopoulos S, Parissis J, Karatzas D, et al. Physical training modulates proinflammatory cytokines and the soluble Fas/soluble Fas ligand system in patients with chronic heart failure. J Am Coll Cardiol 2002;39(4):653-63.

[84] Larsen AI, Lindal S, Aukrust P, et al. Effect of exercise training on skeletal muscle fibre characteristics in men with chronic heart failure. Correlation between skeletal muscle alterations, cytokines, and exercise capacity. Int J Cardiol 2002;83:25-32.

[85] LeMaire JP, Harris S, Fox KA, et al. Change in circulating cytokines after 2 forms of exercise training in chronic stable heart failure. Am Heart J 2004;147(1):100-5.

[86] Conraads V, Bosmans J, Vrints C. Chronic heart failure: an example of systemic chronic inflammatory disease resulting in cachexia. Int J Cardiol 2002;85(1):33-8.

[87] Ennezat PV, Malenddowicz SL, Testa M, et al. Physical training in patients with chronic heart failure enhances the expression of genes encoding antioxidative enzymes. J Am Coll Cardiol 2001;38:194-8.

[88] Shephard RJ. Cytokine responses to physical activity, with particular reference to IL-6: sources, actions, and clinical implications. Crit Rev Immunol 2002;22(3):165-82. 
[89] Goldhammer E, Tanchilevitch A, Maor I, et al. Exercise training modulates cytokine activity in coronary heart disease patients. Int J Cardiol 2005;100(1):93-9.

[90] Smith DA, Irving SD, Sheldon J, et al. Serum levels of the anti-inflammatory cytokine interleukin-10 are decreased in patients with unstable angina. Circulation 2001;104(7): $756-61$.

[91] Maltais F, LeBlanc P, Simard C, et al. Skeletal muscle adaptation to endurance training in patients with COPD. Am J Respir Crit Care Med 1996;154:442-7.

[92] Couillard A, Maltais F, Saey D, et al. Exercise-induced quadriceps oxidative stress and peripheral muscle dysfunction in patients with chronic obstructive pulmonary disease. Am $\mathbf{J}$ Respir Crit Care Med 2003;167(12):1664-9.

[93] Belman MJ, Kendregan BA. Exercise training fails to increase skeletal muscle enzymes in patients with chronic obstructive pulmonary disease. Am Rev Res Dis 1981;123(3):256-61.

[94] Tamura DY, Moore EE, Partrick DA, et al. Acute hypoxemia in humans enhances the neutrophil inflammatory response. Shock 2002;17(4):269-74.

[95] Cummins EP, Taylor CT. Hypoxia-responsive transcription factors. Pflugers Arch: European Journal of Physiology 2005;450:363-71.

[96] Koechlin C, Maltais F, Saey D, et al. Hypoxaemia enhances peripheral muscle oxidative stress in COPD. Thorax 2005;60:834-41.

[97] Koechlin C, Couillard A, Cristol JP, et al. Does systemic inflammation trigger local exercise-induced oxidative stress in COPD? Eur Respir J 2004;23(4):538-44.

[98] Barbeau P, Woods KF, Ramsey LT, et al. Exercise in sickle cell anemia: effect on inflammatory and vasoactive markers. Endothelium 2001;8(2):147-55.

[99] Cadet P, Zhu W, Mantione K, et al. Cyclic exercise induces anti-inflammatory signal molecule increases in the plasma of Parkinson's patients. Int J Mol Med 2003;12(4):485-92.

[100] Bearne LM, Scott DL, Hurley MV. Exercise can reverse quadriceps sensorimotor dysfunction that is associated with rheumatoid arthritis without exacerbating disease activity. Rheumatology 2002;41(2):157-66.

[101] Baldwin KM. Research in the exercise sciences: where do we go from here? J Appl Physiol 2000;88:332-6.

[102] Neviere R, Mathiew D, Chagnon JL. Skeletal muscle microvascular blood flow and oxygen transport in patients with severe sepsis. Am J Respir Crit Care Med 1996;153:191-5. 